\title{
A Secure and Optimally Efficient Multi-Authority Election Scheme
}

\author{
Ronald Cramer ${ }^{\star}$ Rosario Gennaro ${ }^{\star \star}$ Berry Schoenmakers ${ }^{\star \star \star}$
}

\begin{abstract}
In this paper we present a new multi-authority secret-ballot election scheme that guarantees privacy, universal verifiability, and robustness. It is the first scheme for which the performance is optimal in the sense that time and communication complexity is minimal both for the individual voters and the authorities. An interesting property of the scheme is that the time and communication complexity for the voter is independent of the number of authorities. A voter simply posts a single encrypted message accompanied by a compact proof that it contains a valid vote. Our result is complementary to the result by Cramer, Franklin, Schoenmakers, and Yung in the sense that in their scheme the work for voters is linear in the number of authorities but can be instantiated to yield information-theoretic privacy, while in our scheme the voter's effort is independent of the number of authorities but always provides computational privacy-protection. We will also point out that the majority of proposed voting schemes provide computational privacy only (often without even considering the lack of information-theoretic privacy), and that our new scheme is by far superior to those schemes.
\end{abstract}

\section{Introduction}

In the cryptographic literature, electronic voting protocols are known as the prime examples of secure multi-party computations. Many papers have been written on the subject and by now an extensive list of properties and requirements is generally accepted as desirable. We will consider these properties in this paper, among which are privacy, universal verifiability, and various forms of robustness. Recent advancements have also been particularly concerned with the performance aspect. In this paper we will show under which circumstances it is possible to achieve a scheme with optimal performance for large-scale elections, while at the same time keeping the system simple and provably secure.

In considering the performance of elections it is clear that the main consideration should be the effort required of a voter. Indeed, while governments can (and do nowadays) afford a large organizational effort to hold elections, it is mandatory to make the voting protocol as simple and efficient as possible for the voter - who might be participating from home using a PC or a Web TV.

\footnotetext{
* Inst. for Theoretical Comp. Sc., ETH-Z, CH-8092 Zurich, Switzerland. cramer@inf.ethz.ch

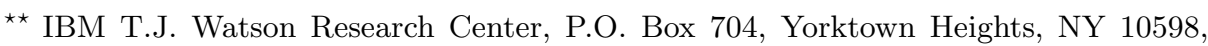
USA. rosario@watson.ibm.com

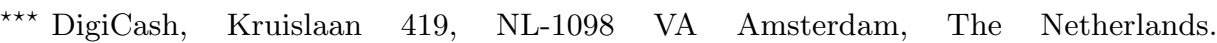
berry@digicash.com
} 
In this paper we present a simple multi-authority election scheme in which the task of the voter is reduced to the bare minimum. Basically, the voter posts a single encrypted message (ballot) accompanied with a proof that it contains a valid vote. For security parameter $k$, the size of the ballot as well as of its proof of validity is $O(k)$ bits. Moreover, due to the homomorphic properties of the encryption method used, the final tally is verifiable to any observer of the election, while due to the use of a matching fault-tolerant threshold decryption technique, the individual votes will remain private and the (benign or malign) failure of authorities can be tolerated.

We work in the model set forth by Benaloh et al. [CF85,BY86,Ben87], where the active parties are divided into $l$ voters $V_{1}, \ldots, V_{l}$ and $n$ tallying authorities (talliers) $A_{1}, \ldots, A_{n}$. To achieve universal verifiability all parties have access to a so-called bulletin board. A bulletin board is like a broadcast channel with memory to the extent that any party (including passive observers) can see the contents of it, and furthermore that each active participant can post messages by appending the message to her own designated area. No party can erase anything from the bulletin board.

In this model, voters cast their votes by posting ballots to the bulletin board. The ballot does not reveal any information on the vote itself but it is ensured by an accompanying proof that the ballot indeed contains a valid vote and nothing else. Due to a homomorphic property of the ballots, the final tally ("sum" of all votes) can be obtained and verified (by any observer) against the "product" of all submitted ballots. This ensures universal verifiability.

Although we are emphasizing the application of our scheme to large-scale elections, it is also suitable for small-scale elections such as boardroom elections. In the latter case it is even conceivable that each voter plays the role of tallying authority as well; a PC network will suffice as computing platform.

\subsection{Computational versus information-theoretic privacy}

By far, the majority of election protocols that support some level of verifiability (either universal or limited to voters, who can check their own vote) merely provide computational protection of the voter's privacy. For example, the schemes presented by Benaloh et al. [CF85,BY86,Ben87,BT94] all rely on the so-called $r$ th residuosity assumption. Once this assumption is broken (e.g., when the public modulus is factorized), the content of each individual ballot can be decrypted. Similarly, schemes using anonymous channels or mixes [Cha81] usually rely on computational assumptions. By recovering the private keys of the mixes, an adversary is able to "open" all ballots posted to the first mix. For example, the scheme of [SK95] relies on the difficulty of computing discrete logs, both for the secrecy of the mixes' private keys and for the contents of the ballots.

The extent to which the lack of information-theoretic privacy is harmful may be difficult to estimate. For instance, it is hard to predict what happens if fiftyyear old votes of a U.S. president are published - although breaking the encryption methods for the currently widely used security parameters will probably be much more harmful.

Whither democracy, from a cryptographic standpoint it is necessary to determine the limits for computational and information-theoretic privacy. As an aside 
we note that the mere use of multiple authorities can be considered a condition as well. Indeed, election protocols have been proposed that try to eliminate this condition, e.g., see [PW92], but the methods used still require conditions regarding the channels connecting the participants. Since in our case the bulletin board is implemented from multiple servers anyway, and it is seen as a necessary primitive for achieving universal verifiability, we will not consider eliminating the use of a distributed tallying authority. Yet, to some extent we will take into account that authorities may be compromised over time, see below.

\subsection{Our contributions}

In this paper we will see how far one can go if computational privacy is the goal. For computational privacy it suffices to assume a public broadcast channel (bulletin board) as communication model. To make an election scheme informationtheoretically secure, it is generally believed that private channels between voters and authorities are required. In Section 6.1 we will look into this aspect.

The main result of this paper is a fair election scheme in which the complexity of the voter's protocol is linear in the security parameter $k$-hence optimal. This comprises the computational as well as the communication complexity (in bits). The voter needs to communicate only $O(k)$ bits and to perform $O(k)$ modular multiplications. ${ }^{1}$ Moreover, the dominating factor for the work of an authority is $O(l k)$. Compared to the scheme of [CFSY96], we thus achieve a reduction of the work for each participant by a factor of $n$.

In the new scheme, the voter just sends a particular ElGamal encryption of the vote plus a proof that it indeed contains a valid vote. The proof prevents the voters from casting bogus ballots, and should be such that no information whatsoever leaks about the actual vote contained in a ballot. The crux is to keep this proof $O(k)$, and here we follow the approach of [CFSY96]. We will need a novel application of the technique of [CDS94] for constructing efficient witness hiding protocols. The resulting proof of validity is a little bit more complicated than in [CFSY96], but still requires only a few modular exponentiations. A proof of knowledge similar to our proof of validity has been used by Chen and Pedersen to construct efficient group signatures [CP95].

Unlike previous schemes based on Benaloh's approach, however, we will achieve robustness w.r.t. faulty authorities without increasing the work for the voter. To this end, we will employ fault-tolerant threshold cryptosystems instead of (verifiable) secret sharing schemes. In our case there will be only one public key for which the matching private key is shared among the authorities using threshold cryptography techniques (see [Des94] for a survey.) The voter posts the ballot encrypted with the public key of the authorities. The private key is never reconstructed, and only used implicitly when the authorities cooperate to decrypt the final tally. The correctness of the decryption will be assured, even in the presence of malicious authorities.

Apart from achieving a strong set of properties, three major achievements of our scheme are: (i) The work required of the voter is minimal. Compared to [CFSY96] the work is reduced by a multiplicative factor of $n$. Although $n$ is

\footnotetext{
${ }^{1}$ Throughout, we will take a modular multiplication of two $O(k)$ sized numbers as our unit of work.
} 
usually much smaller than $k$, this is still a substantial gain in practice. The work for the authorities and observers is reduced accordingly. (ii) The protocol for the voter remains the same even if $n$ is variable. Usually $n$ grows with the desired security of the scheme (the more authorities the less potential that an adversary can corrupt, say, half of them). Using our protocol this growth is "transparent" to the user. (iii) As a bonus, the new scheme can easily be extended using techniques for proactive threshold cryptosystems [HJJ+97] to leave the system (and its keys) in place for a really long time without fearing that the secret key gets compromised (see Section 6.3).

The security of the main scheme presented in the paper is related to the difficulty of the discret log problem. In Section 5 we describe an alternative construction related to the hardness of factoring. Finally, in Section 4 we show how our approach can be extended to more general classes of elections, and in Section 6.2 we consider the issue of receipt-free or incoercible elections and discuss the relevance of our paper in this area.

\section{The building blocks}

\subsection{Bulletin board}

The communication model required for our election scheme is best viewed as a public broadcast channel with memory, which is called a bulletin board. All communication through the bulletin board is public and can be read by any party (including passive observers). No party can erase any information from the bulletin board, but each active participant can append messages to its own designated section.

To make the latter requirement publicly verifiable, we assume that digital signatures are used to control access to the various sections of the bulletin board. Here we may take advantage of any public-key infrastructure that is already in place. Also note that by postulating that each participant can indeed append messages to its section, it is implicitly assumed that denial-of-service attacks are excluded. This property is realized by designing the bulletin board as a set of replicated servers implementing Byzantine agreement, for instance, such that access is never denied as long as at most a third of the servers is compromised. Reiter's work on the Rampart system shows that this can be done in a secure and practical way (see, e.g., [Rei94,Rei95]).

\subsection{ElGamal cryptosystem}

Our election scheme relies on the ElGamal cryptosystem [DH76,ElG85]. It is well-known that the ElGamal cryptosystem works for any family of groups for which the discrete logarithm is considered intractable. Part of the security of the scheme actually relies on the Diffie-Hellman assumption, which implies the hardness of computing discrete logarithms [DH76]. Although all our constructions can easily be shown to work in this general discrete log setting, we will present our results for subgroups $G_{q}$ of order $q$ of $\mathbb{Z}_{p}^{*}$, where $p$ and $q$ are large primes such that $q \mid p-1$. Other practical families can be obtained for elliptic curves over finite fields. 
We will now briefly describe the ElGamal cryptosystem, where the primes $p$ and $q$ and at least one generator $g$ of $G_{q}$ are treated as system parameters. These parameters as well as other independent generators introduced in the sequel should be generated jointly by (a designated subset) of the participants. This can be done by letting the participants each run a copy of the same probabilistic algorithm, where the coinflips are generated mutually at random.

The key pair of a receiver in the ElGamal cryptosystem consists of a private key $s$ (randomly chosen by the receiver) and the corresponding public key $h=g^{s}$, which is announced to the participants in the system.

Given a message $m \in G_{q}$, encryption proceeds as follows. The sender chooses a random $\alpha \in \mathbb{Z}_{q}$, and sends the pair $(x, y)=\left(g^{\alpha}, h^{\alpha} m\right)$ as ciphertext to the receiving party. To decrypt the ciphertext $(x, y)$ the receiver recovers the plaintext as $m=y / x^{s}$, using the private key $s$.

\subsection{Robust threshold ElGamal cryptosystem}

The object of a threshold scheme for public-key encryption is to share a private key among a set of receivers such that messages can only be decrypted when a substantial set of receivers cooperate. See [Des94] for a survey. The main protocols of a threshold system are (i) a key generation protocol to generate the private key jointly by the receivers, and (ii) a decryption protocol to jointly decrypt a ciphertext without explicitly reconstructing the private key. For the ElGamal system described above, solutions for both protocols have been described by Pedersen [Ped91,Ped92], also taking robustness into account.

Key generation As part of the set-up procedure of the election scheme, the authorities will execute a key generation protocol due to Pedersen [Ped91]. The result of the key generation protocol is that each authority $A_{j}$ will possess a share $s_{j} \in \mathbb{Z}_{q}$ of a secret $s$. The authorities are committed to these shares as the values $h_{j}=g^{s_{j}}$ are made public. Furthermore, the shares $s_{j}$ are such that the secret $s$ can be reconstructed from any set $\Lambda$ of $t$ shares using appropriate Lagrange coefficients, say:

$$
s=\sum_{j \in \Lambda} s_{j} \lambda_{j, \Lambda}, \quad \lambda_{j, \Lambda}=\prod_{l \in \Lambda \backslash\{j\}} \frac{l}{l-j} .
$$

This is exactly as in Shamir's $(t, n)$-threshold secret sharing scheme [Sha79]. The public key $h=g^{s}$ is announced to all participants in the system. Note that no single participant learns the secret $s$, and that the value of $s$ is only computationally protected. ${ }^{2}$

Decryption To decrypt a ciphertext $(x, y)=\left(g^{\alpha}, h^{\alpha} m\right)$ without reconstructing the secret $s$, the authorities execute the following protocol:

1. Each authority $A_{j}$ broadcasts $w_{j}=x^{s_{j}}$ and proves in zero-knowledge that

$$
\log _{g} h_{j}=\log _{x} w_{j}
$$

${ }^{2}$ The private channels assumed in Pedersen's key generation protocol may be implemented using public key encryption and the bulletin board. This suffices for computational security. 


$$
\begin{array}{ccc}
\begin{array}{c}
\text { Prover } \\
{\left[(x, y)=\left(g^{\alpha}, h^{\alpha}\right)\right]} \\
w \in \mathbb{Z}_{q}
\end{array} & \text { Verifier } \\
(a, b) \leftarrow\left(g^{w}, h^{w}\right) & \\
r \leftarrow w+\alpha c & \stackrel{a, b}{c} c \in_{R} \mathbb{Z}_{q} \\
r & g^{r} \stackrel{?}{=} a x^{c} \\
h^{r} \stackrel{?}{=} b y^{c}
\end{array}
$$

Fig. 1. Proof of knowledge for $\log _{g} x=\log _{h} y$.

2. Let $\Lambda$ denote any subset of $t$ authorities who passed the zero-knowledge proof. By raising $x$ to both sides of equation (1), it follows that the plaintext can be recovered as

$$
m=y / \prod_{j \in \Lambda} w_{j}^{\lambda_{j, \Lambda}} .
$$

Note that step 2 assures that the decryption is correct and successful even if up to $n-t$ authorities are malicious or fail to execute the protocol. The zero-knowledge proof of step 1 will be described in the next section.

\subsection{Proofs of knowledge for equality of discrete logs}

Using the same notation as above, we present proofs of knowledge for the relation $\log _{g} x=\log _{h} y$, whereby a prover shows possession of an $\alpha \in \mathbb{Z}_{q}$ satisfying $x=g^{\alpha}$ and $y=h^{\alpha}$. An efficient protocol for this problem is due to Chaum and Pedersen [CP93], see Figure 1. This protocol is not known to be zero-knowledge or witness hiding. The following result however suffices for our application (see also [CDS94] for definitions of the notions involved).

Lemma 1 The Chaum-Pedersen protocol is a three-move, public coin proof of knowledge for the relation $\log _{g} x=\log _{h} y$. The proof satisfies special soundness, and is special honest-verifier zero-knowledge.

Proof. The protocol inherits its properties from the underlying Schnorr protocol [Sch91]. Special soundness holds because from two accepting conversations with the same first move $(a, b, c, r)$ and $\left(a, b, c^{\prime}, r^{\prime}\right), c \neq c^{\prime}$, a witness $w=\frac{r-r^{\prime}}{c-c^{\prime}}$ can be extracted satisfying $x=g^{w}$ and $y=h^{w}$. Honest-verifier zero-knowledge holds because, for random $c$ and $r$ we have that $\left(g^{r} x^{-c}, h^{r} y^{-c}, c, r\right)$ is an accepting conversation with the right distribution. Since the challenge $c$ can be chosen freely, we also have special honest-verifier zero-knowledge.

Notice that the above protocol is zero-knowledge only against the honest verifier, but this suffices for our purpose (see, e.g., [Cha91] for an efficient zeroknowledge protocol). Indeed, jumping ahead a little, in order to make our protocols non-interactive, the verifier will be implemented using either a trusted source of random bits (a beacon as in [Rab83,Ben87]) or using the Fiat-Shamir 
heuristic [FS87] which requires a hash function. In the latter case security is obtained for the random oracle model.

\subsection{Homomorphic encryption}

Homomorphic encryption schemes form an important tool for achieving universally verifiable election schemes. A general definition of the notion is as follows. Let $\mathcal{E}$ denote a probabilistic encryption scheme. Let $M$ be the message space and $C$ the ciphertext space such that $M$ is a group under operation $\oplus$ and $C$ is a group under operation $\otimes$. We say that $\mathcal{E}$ is a $(\oplus, \otimes)$-homomorphic encryption scheme if for any instance $E$ of the encryption scheme, given $c_{1}=E_{r_{1}}\left(m_{1}\right)$ and $c_{2}=E_{r_{2}}\left(m_{2}\right)$, there exists an $r$ such that

$$
c_{1} \otimes c_{2}=E_{r}\left(m_{1} \oplus m_{2}\right)
$$

Homomorphic encryption schemes are important to the construction of election protocols. If one has a $(+, \otimes)$ scheme, then if $c_{i}$ are the encryptions of the single votes, by decrypting $c=c_{1} \otimes \ldots \otimes c_{m}$ one obtains the tally of the election, without decrypting single votes.

The ElGamal cryptosystem as presented above already satisfies this definition, where the message space is $G_{q}$ with multiplication modulo $p$ as group operation, and the ciphertext space is $G_{q} \times G_{q}$ with componentwise multiplication modulo $p$ as group operation. Namely, given an ElGamal encryption $\left(x_{1}, y_{1}\right)$ of $m_{1}$ and an ElGamal encryption $\left(x_{2}, y_{2}\right)$ of $m_{2}$, we see that $\left(x_{1} x_{2}, y_{1} y_{2}\right)$ is an ElGamal encryption of $m_{1} m_{2}$.

For the reasons sketched above however, we need to take this one step further to a homomorphic scheme with addition as group operation for the message space. That is, instead of $G_{q}$, our message space will be $\mathbb{Z}_{q}$ with addition modulo $q$ as group operation. Given a fixed generator $G \in G_{q}$, the encryption of a message $m \in \mathbb{Z}_{q}$ will be the ElGamal encryption of $G^{m}$. The observation is now that, given two such encryptions of $m_{1}$ and $m_{2}$, respectively, the product is an encryption of $m_{1}+m_{2}$ modulo $q$. Notice that for such a scheme decryption involves the computation of a discrete log, which is a hard task in general. Nevertheless it can be done efficiently for "small" messages, as will be the case in our election scheme (see Section 3).

\subsection{Efficient proofs of validity}

In our election each voter will post an ElGamal encryption of either $m_{0}$ or $m_{1}$, where $m_{0}$ and $m_{1}$ denote distinct elements of $G_{q}$. (Later we will consider suitable values for $m_{0}$ and $m_{1}$.) The encryption should be accompanied by a proof of validity that proves that the encryption indeed contains one of these values. Furthermore, the proof should not reveal any information about which one.

Consider an ElGamal encryption of the following form:

$$
(x, y)=\left(g^{\alpha}, h^{\alpha} m\right), \quad \text { with } m \in\left\{m_{0}, m_{1}\right\},
$$




$$
\begin{aligned}
& \begin{array}{c|c}
v=1 & v=-1 \\
\hline \alpha, w, r_{1}, d_{1} \in_{R} \mathbb{Z}_{q} & \alpha, w, r_{2}, d_{2} \in_{R} \mathbb{Z}_{q}
\end{array} \\
& x \leftarrow g^{\alpha} \quad x \leftarrow g^{\alpha} \\
& y \leftarrow h^{\alpha} G \quad y \leftarrow h^{\alpha} / G \\
& a_{1} \leftarrow g^{r_{1}} x^{d_{1}} \quad \begin{array}{l}
a_{1} \leftarrow g^{w} \\
b_{1} \leftarrow h^{r_{1}}(y G)^{d_{1}}
\end{array} \\
& \begin{array}{l|l}
b_{1} \leftarrow h^{r_{1}}(y G)^{d_{1}} & b_{1} \leftarrow h^{w} \\
a_{2} \leftarrow g^{w} & a_{2} \leftarrow g^{r_{2}} x^{d_{2}}
\end{array}
\end{aligned}
$$

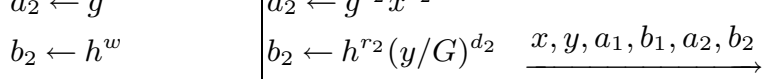

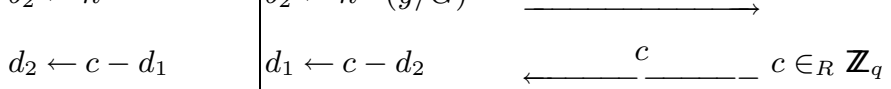

$$
\begin{aligned}
& r_{2} \leftarrow w-\alpha d_{2} \quad r_{1} \leftarrow w-\alpha d_{1} \quad-\quad d_{1}, d_{2}, r_{1}, r_{2} \longrightarrow ? \stackrel{?}{=} d_{1}+d_{2} \\
& a_{1} \stackrel{?}{=} g^{r_{1}} x^{d_{1}} \\
& b_{1} \stackrel{?}{=} h^{r_{1}}(y G)^{d_{1}} \\
& a_{2} \stackrel{?}{=} g^{r_{2}} x^{d_{2}} \\
& b_{2} \stackrel{?}{=} h^{r_{2}}(y / G)^{d_{2}}
\end{aligned}
$$

Fig. 2. Encryption and Proof of Validity of Ballot $(x, y)$

where the prover knows the value of $m$. To show that the pair $(x, y)$ is indeed of this form without revealing the value of $m$ boils down to a witness indistinguishable proof of knowledge of the relation given by:

$$
\log _{g} x=\log _{h}\left(y / m_{0}\right) \quad \vee \quad \log _{g} x=\log _{h}\left(y / m_{1}\right) .
$$

The prover either knows a witness for the left part or a witness for the right part (but not both at the same time), depending on the choice for $m$.

By the techniques of [CDS94], we can now immediately obtain a very efficient witness indistinguishable proof of knowledge for the above relation. To prove either of the two equalities we have the efficient proof of knowledge by Chaum and Pedersen, described above, for which we have prepared Lemma 1. On account of this lemma, we have that the protocol exactly satisfies the conditions for the construction of [CDS94]. See Figure 2 for a preview of the protocol, as it is used in the election scheme of the next section.

\section{Multi-authority election scheme}

Given the primitives of the previous section we now assemble a simple and efficient election scheme. The participants in the election protocol are $n$ authorities $A_{1}, \ldots, A_{n}$ and $l$ voters $V_{1}, \ldots, V_{l}$. Recall that the requirements for a ballot are that it must contain a vote in an unambiguous way such that (i) votes accumulate when ballots are aggregated, and (ii) the proof of validity shows that a 
ballot contains either a yes-vote or a no-vote, without revealing any information on which of the two is the case.

To show that the same masking technique as in [SK94,CFSY96] can be used, we instantiate the scheme of Section 2.6 with $m_{1}=G$ and $m_{0}=1 / G$, where $G$ is a fixed generator of $G_{q}$. Thus a ballot is prepared as an ElGamal encryption of the form $(x, y)=\left(g^{\alpha}, h^{\alpha} G^{b}\right)$ for random $b \in_{R}\{1,-1\}$, and the corresponding proof of knowledge is depicted in Figure 2. To cast a ballot the voter posts an additional number $e \in\{1,-1\}$ such that $v=b e$ is equal to the desired vote. Alternatively, voters may adapt the precomputed values before sending the ballot out, i.e., precompute $(x, y)$ and then post $\left(x^{e}, y^{e}\right)$.

In order to make vote casting non-interactive we compute the challenge $c$ as a hash value of the first message of the proof. In this case security is retained in the random oracle model, but some care is required to prevent vote duplication. Each challenge must be made voter-specific (see [Gen95]), i.e., the challenge $c$ is computed by voter $V_{i}$ as $H\left(I D_{i}, x, y, a_{1}, b_{1}, a_{2}, b_{2}\right)$, where $I D_{i}$ is a unique public string identifying $V_{i}$.

As part of the initialization the designated parties generate the system parameters $p, q, g, G$, as described in Section 2.2, where we may safely assume that $l<q / 2$ for any reasonable security parameter $k$. Secondly, the authorities execute the robust key generation protocol as described in Section 2.3. The transcripts of these protocol should appear on the bulletin board. Note that this also shows to any observer that indeed $n$ authorities are taken part in the scheme, which is otherwise not visible to the voters.

The main steps of the voting protocol now are, where we assume w.l.o.g. that only correct ballots are cast:

1. Voter $V_{i}$ posts a ballot $\left(x_{i}, y_{i}\right)$ to the bulletin board accompanied by a noninteractive proof of validity.

2. When the deadline is reached, the proofs of validity are checked by the authorities and the product $(X, Y)=\left(\prod_{i=1}^{l} x_{i}, \prod_{i=1}^{l} y_{i}\right)$ is formed.

3. Finally, the authorities jointly execute the decryption protocol of Section 2.3 for $(X, Y)$ to obtain the value of $W=Y / X^{s}$. A non-interactive proof of knowledge is used in Step 1 of the decryption protocol.

We thus get $W=G^{T}$ as a result, where $T$ is equal to the difference between the number of yes-votes and no-votes, $-l \leq T \leq l$. Hence, $T=\log _{G} W$ which is in general hard to compute. However, in our case we can now fully exploit the fact that the number of voters $l$ is relatively small-certainly polynomial in the security parameter! The value of $T$ can be determined easily using $O(l)$ modular multiplications only, by iteratively generating $G^{-l}, G^{-l+1}, G^{-l+2}, \ldots$ (each time using one multiplication) until $W$ is found. Asymptotically, the work does therefore not increase for the authorities (at most two multiplications per voter). Note also that the computation of $\log _{G} W$ may be done by any party because the result is verifiable. ${ }^{3}$

The time and communication complexity of the scheme is as follows. The work for a voter is clearly linear in $k$, independent of the number of authorities.

${ }^{3}$ If this $O(l)$ search method is considered too slow for a large-scale election, Shanks' baby-step giant-step algorithm (see, e.g., [LL90, Section 3.1]) can be applied to find $T$ in $O(\sqrt{l})$ time using $O(\sqrt{l} k)$ bits of storage. 
The work for the authorities is only $O(l k+n k)$ (assuming that the zero-knowledge proof used in step 3 is $O(k)$, hence negligible). Since we may safely assume that the number of voters is larger than the number of authorities, the work for the authorities is actually $O(l k)$. Similarly, the work for an observer who wants to check the outcome of the election is $O(l k)$.

Theorem 2 Under the Diffie-Hellman assumption, our election scheme provides universal verifiability, computational privacy, robustness, and prevents vote duplication.

Actually, parts of this theorem also hold under the discrete log assumption, but for conciseness we are only referring to the Diffie-Hellman assumption (which is required to show that the ElGamal encryptions used do not leak information about the votes). For the non-interactive version of the scheme based on the Fiat-Shamir heuristic, the result holds in the random oracle model.

\section{Extension to multi-way elections}

Instead of offering a choice between two options, it is often required that a choice between several options can be made. There are numerous approaches to tackle this problem. Below, we sketch an approach fow which the size of the ballots does not increase (but the size of the proof of validity does), which again relies on the construction of [CDS94]. To get an election for a 1-out-of- $K$ choice, we simply take $K$ (independently generated) generators $G_{i}, 1 \leq i \leq K$, and accumulate the votes for each option separately. The proof of validity of a ballot $(x, y)$ now boils down to a proof of knowledge of

$$
\log _{g} x=\log _{h}\left(y / G_{1}\right) \quad \vee \quad \cdots \quad \vee \quad \log _{g} x=\log _{h}\left(y / G_{K}\right) .
$$

Since the voter can only generate this proof for at most one generator $G_{i}$, it is automatically guaranteed that the voter cannot vote for more than one option at a time.

The problem of computing the final tally is in general more complicated. After decryption by the authorities, a number $W$ is obtained that represents the final tally, $W=G_{1}^{T_{1}} \cdots G_{K}^{T_{K}}$, where the $T_{i}$ 's form the result of the election. Note that the $T_{i}$ 's are uniquely determined by $W$ in the sense that computation of a different set $T_{i}^{\prime}$ 's satisfying $W=G_{1}^{T_{1}^{\prime}} \cdots G_{K}^{T_{K}^{\prime}}$ would contradict the discrete log assumption, using the fact that the generators $G_{i}$ are independently generated. Since $T_{i} \geq 0$ and $\sum_{i=1}^{K} T_{i}=l$, computation of the $T_{i}$ 's is feasible for reasonable values of $l$ and $K{ }^{4}$

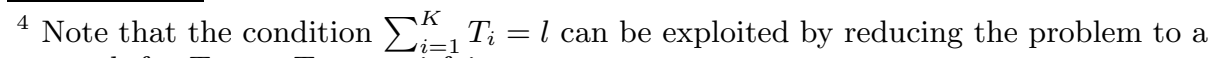
search for $T_{1}, \ldots, T_{K-1}$ satisfying

$$
W / G_{K}^{l}=\left(G_{1} / G_{K}\right)^{T_{1}} \cdots\left(G_{K-1} / G_{K}\right)^{T_{K-1}},
$$

where $T_{i} \geq 0$ and $\sum_{i=1}^{K-1} T_{i} \leq l$. The naive $O\left(l^{K-1}\right)$ method (which checks all possible combinations) can now be improved considerably by a generalization of the baby-step giant-step algorithm of time $O\left(\sqrt{l}^{K-1}\right)$. 


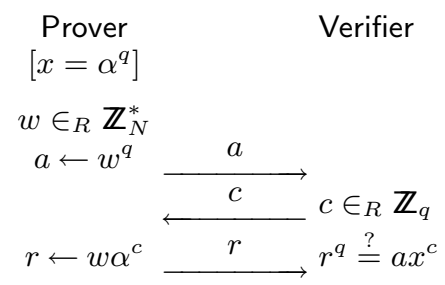

Fig. 3. Proof that $x$ is a $q$-th residue.

\section{Alternative number-theoretic assumption}

To show the generality of our approach we now present a scheme for which the security is related to the difficulty of factoring. Specifically, we present a scheme based on the $q$-th residuosity assumption (as in the original Benaloh schemes). The notion of $q$-th residues is an extension of quadratic residues. A number $x$ is a $q$-th residue modulo $N$ if there exists an $\alpha$ such that $\alpha^{q}=x(\bmod N)$. It is believed to be hard to distinguish between $q$-residues and non $q$-residues.

This suggests the following homomorphic encryption scheme. We present a specific implementation which is suitable to threshold cryptography techniques. The parameters of the scheme are a modulus $N=P Q$, where $P=2 P^{\prime}+1$ and $Q=2 q Q^{\prime}+1$, with $P, Q, P^{\prime}, Q^{\prime}, q$ all large primes. As before, the prime $q$ can thus be assumed to be larger than twice the number of voters $l$. Also the public key must include a fixed number $Y \in \mathbb{Z}_{N}^{*}$ which is not a $q$-th residue modulo $N$.

We will consider messages from $\mathbb{Z}_{q}$. The ciphertext for a message $m$ is now $E_{\alpha}(m)=\alpha^{q} Y^{m}$, where $\alpha \in_{R} Z_{N}^{*}$. As before, decryption is hard, in general, but in our case an exhaustive search for all possible values suffices. The right $m$ is detected when by computing $\left(c Y^{-m}\right)^{\phi(N) / q} \bmod N$ one gets back 1 . Note that $c^{\prime}=c^{\phi(N) / q} \bmod N$ and $Y^{\prime}=\left(Y^{-1}\right)^{\phi(N) / q} \bmod N$ can be computed first, and then test for $c^{\prime} Y^{\prime m}$, where $m$ is selected from all possible messages.

Next we discuss a robust threshold cryptosystem for this setting. Notice that the value $d=\phi(N) / q$ could be considered the secret key of the scheme, and that decryption is carried out by simply computing exponentiations (modulo $N$ ) with exponent $d$. As the setting is very similar to an RSA decryption, we can apply the result of [GJKR96] to obtain an efficient and robust threshold decryption procedure. The result in [GJKR96] holds for RSA moduli which are the product of safe primes (i.e., $P=2 P^{\prime}+1$ and $Q=2 Q^{\prime}+1$ ), but it can easily be extended to work for our specific needs.

The key generation protocol, however, relies on secure multiparty computations as there is no known efficient way to perform a distributed key generation algorithm for factoring based schemes. However, since this task is part of the set-up of the scheme, this may be acceptable as a one-time operation.

Our final task is to construct an efficient proof of validity that shows that a ballot $x$ is correctly formed. This amounts to showing that $x=\alpha^{q} Y^{v}$, for some $\alpha$, with $v \in\{1,-1\}$, hence that either $x / Y$ or $x Y$ is a $q$-th residue. As before, 
Lemma 3 below guarantees the existence of an efficient proof of validity, based on the construction of [CDS94].

Lemma 3 The protocol of Figure 3 is a three-move, public coin proof of knowledge for $r$-th residuosity. The proof satisfies special soundness, and is special honest-verifier zero-knowledge.

Proof. Similar to proof of Lemma 1. Special soundness now holds because for any two accepting conversations $(a, c, r)$ and $\left(a, c^{\prime}, r^{\prime}\right), c>c^{\prime}$, it follows that $\left(r / r^{\prime}\right)^{q}=x^{c-c^{\prime}}$. Since $0<c-c^{\prime}<q$ we have that there exist integers $k, l$ s.t. $\left(c-c^{\prime}\right) k=1+l q$, hence $\left(r / r^{\prime}\right)^{k q}=x^{l q+1}$, which yields $\left(\left(r / r^{\prime}\right)^{k} x^{-l}\right)^{q}=x$.

Theorem 4 Under the q-th residuosity assumption, our election scheme provides universal verifiability, computational privacy, robustness, and prevents vote duplication.

\section{Discussion}

\subsection{Information-theoretically secure elections}

The scheme of [CFSY96] in principle provides information-theoretic protection of the voter's privacy. This is due to the fact that voters post (a number of) information-theoretically hiding commitments to the bulletin board and that these commitments are opened to the authorities using private channels. A general problem with such a solution is that the use of private channels opens the possibility for disputes: on the one hand a dishonest voter may just skip sending a message to an authority, while on the other hand a dishonest authority may claim not to have received a message.

It is therefore worthwhile to limit the possibility for disputes to the set-up process for the election. During the election protocol itself no disputes on the usage of the private channel should be possible. The idea is to use a public broadcast channel (such as a bulletin board) on which the parties post commitments to mutually selected keys. Each pair of parties first agrees on a key using a secure channel. Only if both parties broadcast the same commitment, the set-up of the private channel succeeded. Otherwise, there is a dispute that must be solved at this stage. It is important that (i) the commitment is informationtheoretically hiding and (ii) the encryption method is information-theoretically secure (a one-time pad). More concretely, the two phases are as follows:

Set-up Both parties agree on a mutually at random selected key $K$ and a commitment $B$ on this key. Both parties broadcast a signed copy of the commitment. The key set-up is only succesful if both parties broadcast the same commitment. Disputes in this stage have to be resolved in a procedural way.

Communication To send a message $m$, the sender will broadcast the encryption $E_{K}(m)$ over the public channel. Only the intended receiver is able to recover the message.

Using this method, private channels can be set up from each voter $V_{i}$ to each authority $A_{j}$. Once set up succeeds there can be no dispute on the use of the private channel. Anybody sees if the voter abstains from posting the required 
values to the bulletin board. If what the voter submits consists of incorrect shares, the respective authorities open the commitments to the key so that this fact can be verified. Note that for the scheme of [CFSY96] the use of the private channels is limited to two elements of $\mathbb{Z}_{q}$ per channel.

\subsection{Incoercible protocols}

Receipt-free or incoercible election scheme that have been proposed so far all rely on some form of physical assumption [BT94,NR94,SK95]. The minimal assumption required (as in [SK95]) is the existence of a private channel between the voters and the authorities. These schemes allow a voter to lie about the vote cast even if under coercion, but not up to the level that coercer who exactly prescribe which private random bits the voter must use can be withstood. Indeed given the execution of the protocol the voter will be able to create two different histories of his computations, both consistent with the execution but corresponding to two different votes. All these schemes also require that the authorities are incoercible, or alternatively that voters know which ones have been coerced. Moreover, as pointed out in the previous section, the use of private channels gives rise to disputes. (Another viable approach is to assume that the voters dispose of a tamper-proof encryption box such as a smartcard, but we consider this beyond the scope of this paper.)

Recently, Canetti and Gennaro in [CG96] proved that general secure multiparty computation protocols can be made incoercible without the above assumptions, in particular without assuming untappable channels. Their scheme is based on a new type of encryption called deniable encryption introduced in [CDNO96] that allows a sender to encrypt a bit $b$ in such a way that the resulting ciphertext can be "explained" as either $b$ or $1-b$ to a coercer. The construction in [CG96] works for the general problem of secure multi-party computation; as such it is described in terms of a complete network of communication and the result holds as long as at most half of the players in the network are coerced. For the case of election schemes, the construction of [CG96] can be scaled down to the bulletin board model (thus not requiring communication between voters). In this model all voters can withstand coercion provided the coercer is not able to prescribe the random bits of the voters, and at most half of the authorities can be completely coerced. The complexity of the resulting scheme is high (although polynomial), but opens the door to the search for efficient incoercible schemes.

In order to make our election scheme incoercible (without physical assumptions) we would need a deniable encryption scheme which is (i) homomorphic, (ii) suitable to threshold cryptography techniques. An interesting open problem is thus to construct such a scheme.

\subsection{Proactive Security}

The secrecy of the votes is protected against coalitions of up to $t-1$ authorities. In other words, an attacker must recover $t$ shares of the private key in order to be able to decrypt single votes. This is similar to previous protocols in which the vote is $(t, n)$-shared among the authorities. We note that the use of threshold cryptography instead of secret sharing presents also some advantages in this 
area. Using proactive security techniques (see [HJKY95,HJJ+97,FGMY96]) it is possible to leave the public key of the system in place for a really long time without fearing it being compromised. Indeed, when using proactive schemes the shares of the private key are periodically "refreshed" so that an attacker is forced to recover $t$ shares in one single period of time that can be as short as a day. Both schemes presented in this paper can be made proactive, the discrete-log based one using the techiniques in $\left[\mathrm{HJJ}^{+} 97\right]$ and the factoring one by adapting the work of [FGMY96]. The idea is that the authorities run the key generation protocol every day at midnight, say, but now sharing a zero value. The new shares are added to the old shares of the secret key $s$. The resulting shares still interpolate to $s$ (since the free term of the polynomial is unchanged) but lie on an otherwise different polynomial.

\section{Concluding remarks}

We have shown a very efficient scheme for secure elections based on the discrete log assumption, and a somewhat more complicated scheme based on the $q$-th residuosity assumption. The new schemes satisfy all well-known requirements, except for receipt-freeness. An open problem is to construct efficient incoercible election protocols, preferably without relying on physical assumptions.

In our scheme the work for the voter is minimal and independent of the number of authorities. Election schemes based on the mix channel of [PIK94] also have this property but for several reasons our approach is preferable over those schemes. In mix-based schemes the final tally is computed by somehow decrypting the individual ballots, while in our approach a single decryption of the aggregate of the ballots suffices. In mix-based schemes disrupters may submit invalid ballots which are detected only after decryption has taken place; in our scheme disruption by voters is automatically prevented because of the required proof of validity for ballots. Another important difference is that due to the use of a threshold cryptosystem we achieve robustness in a stronger sense. Indeed in mix-based schemes the failure of a single authority would compromise the whole protocol. In our case we can tolerate malicious behavior of a constant fraction (half) of authorities. Finally, the security of our scheme can be proven from its construction, while some security problems with the schemes of [PIK94,SK95] exist, as shown for instance in [Pfi95,MH96].

We would like to emphasize that the work for the voter is really low. For example, for the discrete $\log$ scheme, we have for $|p|=64$ bytes and $|q|=20$ bytes, that the size of the ballot plus its proof plus a signature on it is only 272 bytes in total. Clearly, this is an order of magnitude better than [CFSY96], which was already two orders of magnitude better than any previous scheme. Furthermore, computation of the ballot and its proof require a few exponentiations only (see Figure 2). A direct consequence of the reduced ballot size is also that the task of verifying the final tally is much simpler.

\section{References}

[Ben87] J. Benaloh. Verifiable Secret-Ballot Elections. PhD thesis, Yale University, Department of Computer Science Department, New Haven, CT, September 
1987.

[BT94] J. Benaloh and D. Tuinstra. Receipt-free secret-ballot elections. In Proc. 26th Symposium on Theory of Computing (STOC'94), pages 544-553, New York, 1994. A.C.M.

[BY86] J. Benaloh and M. Yung. Distributing the power of a government to enhance the privacy of voters. In Proc. 5th ACM Symposium on Principles of Distributed Computing (PODC' '86), pages 52-62, New York, 1986. A.C.M.

[CDNO96] R. Canetti, C. Dwork, M. Naor, and R. Ostrovsky. Deniable encryption, 1996. Manuscript.

[CDS94] R. Cramer, I. Damgård, and B. Schoenmakers. Proofs of partial knowledge and simplified design of witness hiding protocols. In Advances in Cryptology-CRYPTO '94, volume 839 of Lecture Notes in Computer Science, pages 174-187, Berlin, 1994. Springer-Verlag.

[CF85] J. Cohen and M. Fischer. A robust and verifiable cryptographically secure election scheme. In Proc. 26th IEEE Symposium on Foundations of Computer Science (FOCS '85), pages 372-382. IEEE Computer Society, 1985.

[CFSY96] R. Cramer, M. Franklin, B. Schoenmakers, and M. Yung. Multi-authority secret ballot elections with linear work. In Advances in CryptologyEUROCRYPT '96, volume 1070 of Lecture Notes in Computer Science, pages 72-83, Berlin, 1996. Springer-Verlag.

[CG96] R. Canetti and R. Gennaro. Incoercible multiparty computation. In 37th IEEE Symposium on Foundations of Computer Science (FOCS' 96), 1996. To appear.

[Cha81] D. Chaum. Untraceable electronic mail, return addresses, and digital pseudonyms. Communications of the ACM, 24(2):84-88, 1981.

[Cha91] D. Chaum. Zero-knowledge undeniable signatures. In Damgård, editor, Advances in Cryptology-EUROCRYPT' 90 , volume 473 of Lecture Notes in Computer Science, pages 458-464, Berlin, 1991. Springer-Verlag.

[CP93] D. Chaum and T. P. Pedersen. Wallet databases with observers. In Advances in Cryptology-CRYPTO '92, volume 740 of Lecture Notes in Computer Science, pages 89-105, Berlin, 1993. Springer-Verlag.

[CP95] L. Chen and T. P. Pedersen. New group signature schemes. In Advances in Cryptology-EUROCRYPT '94, volume 950 of Lecture Notes in Computer Science, pages 171-181, Berlin, 1995. Springer-Verlag.

[Des94] Y. Desmedt. Threshold cryptography. European Transactions on Telecommunications, 5(4):449-457, 1994.

[DH76] W. Diffie and M. E. Hellman. New directions in cryptography. IEEE Transactions on Information Theory, 22(6):644-654, 1976.

[ElG85] T. ElGamal. A public-key cryptosystem and a signature scheme based on discrete logarithms. IEEE Transactions on Information Theory, IT31(4):469-472, 1985.

[FGMY96] Y. Frankel, P. Gemmell, P. McKenzie, and M. Yung. Proactive RSA, 1996. Manuscript.

[FS87] A. Fiat and A. Shamir. How to prove yourself: Practical solutions to identification and signature problems. In Advances in Cryptology-CRYPTO '86, volume 263 of Lecture Notes in Computer Science, pages 186-194, New York, 1987. Springer-Verlag.

[Gen95] R. Gennaro. Achieving independence efficiently and securely. In Proc. 14th ACM Symposium on Principles of Distributed Computing (PODC' '95), New York, 1995. A.C.M.

[GJKR96] R. Gennaro, S. Jarecki, H. Krawczyk, and T. Rabin. Robust and efficient sharing of RSA functions. In Advances in Cryptology-CRYPTO '96, volume 1109 of Lecture Notes in Computer Science, pages 157-172, Berlin, 1996. Springer-Verlag. 
$\left[\mathrm{HJJ}^{+} 97\right]$ A. Herzberg, M. Jakobsson, S. Jarecki, H. Krawczyk, and M. Yung. Proactive public-key and signature schemes. 4th Annual Conference on Computer and Communications Security, 1997. To appear.

[HJKY95] A. Herzberg, S. Jarecki, H. Krawczyk, and M. Yung. Proactive secret sharing, or: How to cope with perpetual leakage. In Advances in CryptologyCRYPTO '95, volume 963 of Lecture Notes in Computer Science, pages 339-352, Berlin, 1995. Springer-Verlag.

[LL90] A. K. Lenstra and H. W. Lenstra, Jr. Algorithms in number theory. In J. van Leeuwen, editor, Handbook of Theoretical Computer Science, pages 673-715. Elsevier Science Publishers B.V., Amsterdam, 1990.

[MH96] M. Michels and P. Horster. Some remarks on a receipt-free and universally verifiable mix-type voting scheme. In Advances in CryptologyASIACRYPT' '94, volume 1163 of Lecture Notes in Computer Science, pages 125-132, Berlin, 1996. Springer-Verlag.

[NR94] V. Niemi and A. Renvall. How to prevent buying of votes in computer elections. In Advances in Cryptology-ASIACRYPT '94, volume 739 of Lecture Notes in Computer Science, pages 141-148, Berlin, 1994. SpringerVerlag.

[Ped91] T. Pedersen. A threshold cryptosystem without a trusted party. In $A d$ vances in Cryptology - EUROCRYPT '91, volume 547 of Lecture Notes in Computer Science, pages 522-526, Berlin, 1991. Springer-Verlag.

[Ped92] T. P. Pedersen. Distributed Provers and Verifiable Secret Sharing Based on the Discrete Logarithm Problem. PhD thesis, Aarhus University, Computer Science Department, Aarhus, Denmark, March 1992.

[Pfi95] B. Pfitzmann. Breaking an efficient anonymous channel. In Advances in Cryptology-EUROCRYPT '94, volume 950 of Lecture Notes in Computer Science, pages 332-340, Berlin, 1995. Springer-Verlag.

[PIK94] C. Park, K. Itoh, and K. Kurosawa. Efficient anonymous channel and all/nothing election scheme. In Advances in Cryptology-EUROCRYPT '93, volume 765 of Lecture Notes in Computer Science, pages 248-259, Berlin, 1994. Springer-Verlag.

[PW92] B. Pfitzmann and M. Waidner. Unconditionally untraceable and faulttolerant broadcast and secret ballot election. Hildesheimer informatikberichte, Institut für Informatik, May 1992.

[Rab83] M. Rabin. Transaction protection by beacons. Journal of Computer and System Sciences, 27(2):256-267, 1983.

[Rei94] M. Reiter. Secure agreement protocols: Reliable and atomic group multicast in Rampart. 2nd ACM Conference on Computer and Communications Security, Fairfax, November 1994.

[Rei95] M. Reiter. The Rampart toolkit for building high-integrity services. In Theory and Practice in Distributed Systems, volume 938 of Lecture Notes in Computer Science, pages 99-110, Berlin, 1995. Springer-Verlag.

[Sch91] C. P. Schnorr. Efficient signature generation by smart cards. Journal of Cryptology, 4(3):161-174, 1991.

[Sha79] A. Shamir. How to share a secret. Communications of the ACM, 22(11):612613, 1979.

[SK94] K. Sako and J. Kilian. Secure voting using partially compatible homomorphisms. In Advances in Cryptology - CRYPTO '94, volume 839 of Lecture Notes in Computer Science, pages 411-424, Berlin, 1994. Springer-Verlag.

[SK95] K. Sako and J. Kilian. Receipt-free mix-type voting scheme - a practical solution to the implementation of a voting booth. In Advances in CryptologyEUROCRYPT '95, volume 921 of Lecture Notes in Computer Science, pages 393-403, Berlin, 1995. Springer-Verlag. 